Résumés des conférences et travaux

Histoire du droit public, de l'Ancien Régime à nos jours

Conférences des années 2010-2011 et 2011-2012

\title{
François Monnier
}

\section{OpenEdition \\ Journals}

\section{Édition électronique}

URL : https://journals.openedition.org/ashp/1513

DOI : 10.4000/ashp.1513

ISSN : 1969-6310

\section{Éditeur}

Publications de l'École Pratique des Hautes Études

\section{Édition imprimée}

Date de publication : 1 septembre 2013

Pagination : 194-204

ISSN : 0766-0677

\section{Référence électronique}

François Monnier, "Histoire du droit public, de l'Ancien Régime à nos jours », Annuaire de l'École pratique des hautes études (EPHE), Section des sciences historiques et philologiques [En ligne], 144 | 2013, mis en ligne le 07 novembre 2014, consulté le 05 novembre 2021. URL : http:// journals.openedition.org/ashp/1513; DOI : https://doi.org/10.4000/ashp.1513 


\title{
MÉTHODOLOGIE \\ DE LA PROSOPOGRAPHIE À L’ÉPOQUE CONTEMPORAINE
}

\author{
Maître de conférences : $\mathrm{M}^{\text {me }}$ Isabelle PARIzET
}

Programme de l'année 2011-2012 : I. Les sculpteurs de façades et ornemanistes au XIX $X^{e}$. — II. L'exposition universelle de 1878.

Bien que moins marquante que l'exposition de 1867 et moins prestigieuse que ne le sera celle de 1889, l'exposition de 1878 sut être novatrice sur certains points. Le séminaire a surtout analysé la série $\mathrm{F}^{12} 3198$ à 3756 des Archives nationales, les différents rapports ou publications officiels, et les guides et périodiques contemporains [ex. Les Curiosités de l'exposition ou le guide Joanne].

Pour la première fois en 1878, le périmètre de l'exposition s'étendit des deux côtés du fleuve : la Seine faisait partie du décor. Jamais ponts et rives n'avaient été ainsi théâtralisés. Le pont d'Iéna, élargi pour les besoins de la circulation, se voyait doté en son centre de bancs de repos abrités : installés dos à dos, ces sièges en lattes de bois étaient décorés de bacs à fleurs et couverts de deux hampes croisés [Arch. nat., $\mathrm{F}^{12}$ 3234]. Cette halte permettait de jouir du panorama vers l'amont comme vers l'aval. D'autre part, le ballon captif de Giffard et les ascenseurs des tours du Trocadéro donnèrent l'occasion à des centaines de personnes de profiter de vues aériennes, non seulement sur l'Exposition mais sur l'ensemble de Paris. L'objet de la visite ne se limitait donc plus au seul périmètre de la manifestation mais à la ville toute entière. D'ailleurs, Émile Zola nota que le jour de l'inauguration, les rues s'habillèrent de couleurs nationales... Personne ne se souvenait d'avoir vu Paris sous un tel aspect [Cf. « Lettre de Paris. L'ouverture de l'exposition universelle », Le Messager de l'Europe, XXVIII, 12-24 mai 1878]. Un mois plus tard, le 30 juin, Claude Monet fut frappé à son tour par cette mise en spectacle de Paris : dans « La rue Montorgueil» ou « La rue SaintDenis ", la rue, les immeubles et leur perspective sont le décor de la fête. Les drapeaux aux fenêtres y célèbrent la beauté retrouvée de la capitale et réaffirment sa fidélité à la République. En associant la ville à l'Exposition, le Commissariat général exorcisa définitivement les souvenirs des canonnades destructrices de 1870 et des combats de rues de 1871 .

Si l'exposition est « universelle », il est clair que le visiteur ne reste pas patriote de sa seule nation d'origine mais devient « citoyen du monde». Six ans auparavant, Jules Verne avait publié un feuilleton dans Le Temps qui avait favorisé cette évolution: Le Tour du monde en quatre-vingts jours décrivait la magie de l'exotisme, la rapidité des nouvelles voies de communication et l'évolution des techniques. Un autre périple imaginaire avait paru l'année précédente dans un manuel d'apprentissage de lecture : Le Tour de la France par deux enfants présentait au cours d'un périple 
hexagonal aux enfants l'histoire, la géographie, les valeurs morales et les ressources de leur patrie. L'idée de voyage accessible était donc dans les esprits. Or, sans quitter Paris, une seule promenade de $630 \mathrm{~m}$, permettaient aux visiteurs-voyageurs de découvrir rue des Nations quelques grands monuments européens [le portail de Belem], de se laisser surprendre par des architectures inconnues [le minuscule pavillon de Siam] et de concevoir d'autres formes d'habitat [les maisons russes] : l'enfilade pittoresque des façades alternait architecture rurale et urbaine, publique ou domestique, administrative ou religieuse, ancienne ou moderne. Grâce aux différents lieux d'exposition, un même pays put faire connaître divers exemples de son architecture nationale : pour ne prendre qu'un exemple, le Japon y exposa un exemple d'architecture citadine, puis une ferme et un pavillon à thé entourés de plantations et d'animaux locaux au Trocadéro et enfin son stand à l'intérieur du palais du Champ de Mars. De plus, sur l'ensemble de la Section étrangère, la présentation et la démonstration des produits, étaient faites par un personnel local, la plupart du temps en costume national : l'intérêt puis le goût pour l'orientalisme gagnèrent peu à peu le public qui, après avoir été dérouté par « cet ailleurs impénétrable », sut en apprécier quelques objets accessibles au goût européen [cf. tissus, vases]. La bourgeoisie parisienne, lassée des fabrications usuelles devenues populaires à force d'être présentées dans les expositions précédentes, fut fascinée par cet exotisme et par le raffinement des décors qu'elle y découvrit : elle se laissa gagner par l'attrait de l'étranger. Ce gigantesque panorama architectural des nations exposantes connut donc un immense succès : s'il déroutait le visiteur, il l'impressionnait, le charmait et surtout enrichissait son vocabulaire esthétique, architectural et ornemental.

Dans les années qui suivirent cette exposition, l'architecture parisienne se fit d'ailleurs l'écho de cette fascination. Moins de quatre ans plus tard à l'été 1882, les passants découvrirent une curieuse façade à l'angle du faubourg Saint-Honoré [no 4 ] et de la rue Royale $\left(8^{\mathrm{e}}\right)$ : l'immeuble était occupé par le magasin de thés et glaces du fabricant de chocolats Pihan. Il est probable que ni son propriétaire M. Fourier, ni son architecte Victor Guillemin n'avaient fait le voyage vers des contrées aussi lointaines. Par contre, la découverte de l'architecture étrangère à l'exposition avait $\mathrm{du}$ susciter leur intérêt et entrainer une réflexion afin de conformer le décor architectural de leur immeuble avec l'exotisme du produit commercial de la maison : il en résulta une incroyable façade « de style indo-sarrasin » inspirée des photographies rapportées d'Inde par Louis Rousselet [cf. pl. 158, Dict. par noms d'architectes, $1^{\text {re }}$ série, t. III, $\left.\mathrm{n}^{\mathrm{o}} 2410\right]$. Un autre lieu public était en cours de construction à la même époque : commencé en 1881, l'Eden théâtre fut inauguré le 7 janvier 1883. Les deux architectes Albert Duclos et William Klein choisirent pour cette salle élevée rue Boudreau $\left(9^{\mathrm{e}}\right)$ un style d'inspiration nettement indienne : le bâtiment reposant sur de solides colonnes à piliers, l'étage était orné de petites niches et de consoles à têtes d'éléphants. Le toit s'élevait en pyramide étagée à clochetons et son décor de stuc reprenait certains motifs de cachemire [cf. pl. 43, Ibid., t. I]. Ces architectes avaient déjà manifesté en 1876 leur intérêt et leur goût pour l'exotisme en construisant des « thermes turcoromains » au 56, rue Neuve-des-Mathurins à l'angle de la rue Auber [cf. pl. 44-45, Ibid., t. I, $\mathrm{n}^{\mathrm{o}} 1716$ et $\mathrm{t}$. III $\mathrm{n}^{\mathrm{o}} 2728$ ] : ce hammam réunissait salle de massage, piscine de source, étuves à sec (tepidarium et caldarium voûtées en plein cintre), douches, salle de repos et buffet, installés dans des salles éclairées par des vitraux de couleur et 
meublées de divans de marbre et de sofas. Les pièces réservées aux bains étaient couvertes de mosaïques de marbre. Toujours dans cet esprit, un autre établissement fut élevé par Alexandre Marcel en 1887 au 43, rue de Babylone (7e) [cf. Ibid., pl. 126-127, t. III, $\left.\mathrm{n}^{\circ} 3333\right]$ : La Construction Moderne souligne que les progrès de l'hygiène exigeant des établissements balnéaires plus compliqués, on prit modèle sur les thermes antiques et les bains orientaux. L'emploi de la polychromie a su faire ici une æuvre coquette et de bon goût [18 mars 1893, p. 283]. Il ne faut pas oublier que c'est ce même Marcel qui, après 1889, élèvera une « salle des fêtes de style japonisant » pour M. Morin directeur du Bon Marché au 57, rue de Babylone (7e) [Ibid., pl. 128-129]. Quant au Proche-Orient, il exerça lui aussi une sorte d'attraction, mais le plus souvent dans des constructions publiques qui conjuguait architecture républicaine et esthétique rationaliste [cf. l'exemple du ministère des Travaux publics inspiré par l'architecture arabe dans son pavillon].

Si l'Orient était synonyme d'évasion et de voyage insolite, l'Europe fit soudain rêver à son tour : en arpentant la rue des Nations, le visiteur de 1878 découvrait que l'aventure était aux portes de chez lui. Aussi, quelques années plus tard, les façades flamandes se retrouvèrent boulevard Pereire aux n $\mathrm{n}^{\text {os }}$ 93-95 où l'architecte Jean Brisson dessina une lucarne couronné d'un large pignon à redans de style néo-flamand aux deux petits hôtels de M. Webster en 1883 [cf. pl. 82, Ibid., t. I, no 839]. Toujours dans ce quartier, M. Thors, dans son hôtel du 5, rue de Montchanin [actuelle rue JacquesBingen] présenta fin 1880 un décor Renaissance espagnole, qualifié par les contemporains « d'hispano-flamand » qui rappelait ceux exposés par l'Amérique centrale et la Belgique [cf. Ibid., pl. 61, t. II, no 1868].

Avec l'idée du « voyage accessible à tous, » mûrit l'idée de maison démontable, reconstructible au hasard des étapes successives. En 1867, les chalets de MM. Waaser et Bougleux, répondant à la demande des « bourgeois en mal de campagne », avaient connu un vif succès. En 1878, cet établissement exposa une préfabrication industrielle de pièces à assembler : on salua la finition remarquable des assemblages de leur petit pavillon en bois découpés et la qualité de leurs dessins ornementaux. Adaptant au goût français ce que chinois, japonais, russes ou norvégiens avaient pu présenter aux expositions précédentes, sous la direction de l'architecte Eugène Bardon, M. Waaser a su débarrasser ces constructions en bois apparents de cette manie, si mesquine de la découpure et cette fois, on remarque dans son cuvre, très originale du reste, les influences diverses des arts suédois, norvégien, et russe enrichissant la charpente de tradition allemande et la silhouette française de l'ensemble [cf. Ibid., pl. 35-40; Arch. nat., F' 3541 ; Revue gén. de l'architecture, XXXVIII, 1881, col. 163-164].

La présentation des nouveautés en matière de techniques de construction (Classe 66) était un des atouts majeurs des Expositions. Les entrepreneurs parisiens avaient une réputation de dextérité et d'ingéniosité pour leur système d'échafaudage. Habitués de longue date à construire dans des rues peu larges dont ils ne devaient pas bloquer la circulation, ils avaient mis au point des monte-charges installés dans d'étroites cages verticales placées devant la façade : à l'aide de petites chèvres mues par un treuil, cet ingénieux procédé permettait d'élever et de distribuer les matériaux à la hauteur voulue sans empiéter exagérément sur la chaussée. L'emploi récent de puissants appareils 
d'éclairage électrique avait également accru l'activité des chantiers. Des machines fixes [locomobiles, moteurs à gaz ou à air comprimé] et des machines mobiles [excavateurs, pompes, treuils, grues, mais aussi scies à vapeur, machines à dresser, tailler ou moulurer la pierre, bétonnières] facilitaient les travaux des terrassiers, maçons, appareilleurs et tailleurs de pierre. Circulant dans les deux sens et sur des plaques tournantes installées suivant les besoins du service, des wagonnets à bascule tractés par des machines ou des câbles sur d'étroites voies ferrées ménageaient hommes et chevaux. Cette ordonnance plus rationnelle du chantier et cette mécanisation étendue permettaient un emploi plus efficace de l'outillage et des forces mécaniques, et une économie importante en temps et personnel.

De façon fort judicieuse, la construction des deux palais illustrait les deux grands types de construction employés à l'époque : celle du Trocadéro en maçonnerie classique reprenait une pratique traditionnelle confirmée mais gourmande en main d'œuvre, celle du Champ de Mars en structure métallique, une technologie moderne mais tributaire des lenteurs de la production industrielle. Le rapporteur François Husson estima que les constructions métalliques n'offrent rien de nouveau car tous ces abris ne sont que provisoires et les divers ingénieurs chargés de les élever ont du songer tout d'abord au moyen d'en tirer parti une fois l'exposition terminée [Eugène Lacroix, Études sur l'exposition universelle de 1878, IV, p. 330]. La nécessité de trouver facilement des acquéreurs avait primé sur l'originalité ou la singularité du projet. Les exigences de rapidité de construction, d'économie budgétaire, la production massive et la complexité du programme requerraient l'emploi de procédés efficaces et bon marché. Chaque métal y avait son rôle propre, le fer assurant les supports verticaux, la fonte les horizontaux et le décor [cf. pavillon de la Ville de Paris, galerie intérieure du Champ de Mars, pavillon du ministère des Travaux publics, etc.].

Dans la Section étrangère, la rue des Nations proposait un parcours totalement inhabituel : une demeure en bois sculpté danoise y côtoyait la façade d'un palais milanais et l'entrée d'une maison de campagne japonaise. Tous les pays étrangers exposants y étaient représentés au devant de leur section, sur le promenoir à ciel ouvert qui les longeait : chaque tronçon de façade comportait un développement égal à la largeur de la surface affectée dans l'exposition à la nation concernée. Ces façades d'une largeur de 8 à 60 mètres n'en avaient en moyenne que cinq de profondeur et n'étaient donc qu'un simple décor dressé dans des matériaux légers et économiques.

Le palais du Trocadéro répondait à un projet spécifique : depuis 1864, une « proposition d'orphéon » circulait afin que Paris ait un vrai opéra populaire. Le projet initial place du Château-d'Eau ayant été abandonné, la colline de Chaillot s'imposa rapidement tant par sa position exceptionnelle en face du Champ de Mars que par son relief. Le cahier des charges souhaitait un lieu de fêtes publiques où concerts, fêtes, bals, feux d'artifice pourraient se dérouler et réunir la population parisienne [Arch. nat., $\mathrm{F}^{12}$ 3218]. Sa fonction ne devait pas se limiter à une salle de concerts, ni à un musée mais à un ensemble de salles d'exposition et de congrès, de promenades couvertes, et de points de vue sur toute la capitale. Les hebdomadaires contemporains attestent combien le but défini fut atteint: toutes les fêtes organisées autour de l'évènement n'eurent pas lieu au Champ de Mars mais au Trocadéro et la majorité des objetssouvenirs édités pour l'occasion le représentaient [cf. assiettes, éventails, encriers, 
mouchoirs]. D'autre part, l'architecture même du palais soulignait cette double ouverture intérieure/extérieure par la multiplication d'arcades, baies, galeries, balustrades [pour rejoindre rapidement les différents lieux et/ou pour profiter de sa position géographique]. Il était aisément accessible des quais, des jardins, ou de la place du Roide-Rome [pour les visiteurs venant de Passy ou de l'Étoile] et on y jouissait d'une vue spectaculaire tant sur l'exposition que sur la partie sud de la capitale.

D'autre part, plusieurs matériaux furent améliorés, explorés ou découverts. La Classe 20 proposa un large catalogue de céramique [matières obtenues après cuisson de l'argile soit terre cuite, grès, faïence, porcelaine, etc.]. Leur application à l'ornementation architecturale s'était considérablement développée depuis l'exposition de Londres en 1851 où le comte de Laborde avait salué leur résistance à la pollution et aux intempéries. Associant nature, art et industrie, ces produits étaient de plus en plus appréciés pour leur coût économique et leur rôle décoratif : les rationalistes comme Anatole de Baudot ou Paul Sédille les recommandaient estimant qu'ils répondaient parfaitement à « l'exigence d'utilisation logique des matériaux, par leurs qualités, leur nécessité architecturale et leur fonction décorative ». Les variations de cuisson et de coloration et la comparaison avec les produits étrangers permirent de nouvelles applications. Dans la section française, des fabriques historiques comme celle des frères Virebent de Toulouse, les Peyrusson de Limoges, ou les faïenceries de Choisy-le-Roi de M. Boulenger exposèrent aux côtés d'établissements plus récents comme ceux d'Émile Muller d'Ivry ou les ateliers parisiens de Jules Paul Lœbnitz ou de Théodore Deck : en dehors des briques et des tuiles ornementées, furent présentés chéneaux de faitages, garnitures de rives et de pignons, roses, médaillons et frises. Des effets décoratifs furent obtenus par alternance de taille, de motifs ou de couleur [cf. pavillons du Danemark ou des Travaux publics], leur association avec d'autres éléments de terre cuite [cf. le petit pavillon de la maison Doulton visible actuellement avenue Pascal à Maisons Laffitte], ou insertion d'éléments d'architecture en cette matière [les hollandais avaient élevé un portail roman où alternaient motifs simples ou moulurés selon l'emplacement]. 18 médailles d'or furent décernées aux français : une aux deux frères Hippolyte et Jean Baptiste Boulenger [Hippolyte reçut également la croix de Légion d'honneur], une à Muller [qui venait de construire l'usine de M. Menier à Noisiel avec l'architecte Jules Saulnier], deux autres à la société d'Étienne Gillet et à celle de Lœbnitz pour la porte monumentale exécutée sous le porche des Beaux-Arts. Les Virebent frères et fils se virent attribuer une médaille d'argent pour encourager ces infatigables chercheurs qui s'inspirent des bonnes traditions du passé pour donner un grand caractère à leurs œuvres, ainsi que Damousse et Léon Parvillée : ce dernier très épris de décor oriental avait déjà décoré à l'Exposition de 1867 un pavillon pour la Turquie et publié en 1874 avec Viollet-le-Duc, "Architecture et décorations turques au XV vime siècle » : cette fois, sous la direction de l'architecte Charles Wable, il réalisa le décor du pavillon algérien en faïences polychromes et arabesques de couleurs vives [cf. Arch. nat. : $\mathrm{F}^{12} 3227$ et $\mathrm{F}^{12}$ 3338]. Ces différents fabricants présentèrent avec l'Union céramique et chaufournière [dont la devise était Union dans le travail, Progrès dans la céramique], toutes sortes de terres cuites, modestes ou somptueuses, où ils ont fait preuve d'idées grandioses et patriotiques en introduisant dans l'architecture leur art merveilleux. L'architecture de ce petit pavillon polygonal avait été confiée à 
Marcel Deslignières. Il ne s'agissait pas pour la société d'y apparaître comme « fabricant d'ornements » mais comme « industriel du bâtiment » à part entière : ainsi, un escalier en terre cuite y fut exposé, alors que la malléabilité de ce matériau semblait à première vue inadéquate pour cette destination. D'autres pièces moulées, tournées à la machine ou à la main [statues, culs de lampe, consoles, balustres, carreaux, vases, fontaines] côtoyaient crêtes, tuyaux ou briques creuses et prouvaient que ces matériaux pouvaient parfaitement s'inscrire dans « l'art du bâtiment »

La palette variée de ce matériau économique séduisit les architectes parisiens : que ce soit Eugène Flamant en 1879 pour l'hôtel modeste de M. Bellet rue d'Offémont [cf. pl. 75, Dict., t. II], ou Jules Février en février 1881 pour l'incroyable pastiche Renaissance commandé par le banquier Gaillard place Malesherbes (17e) [Ibid., pl. 74]. Sans rester cantonné aux bâtiments utilitaires [les communs élevés par Frantz Jourdain avenue du bois de Boulogne, cf. La Construction moderne, 18 juin 1892, p. 437], il conquit également les artistes comme le peintre Aimé Morot pour son hôtel-atelier construit par Émile Jeandel, 11, rue Weber (16 ${ }^{\mathrm{e}}$ ) en 1885-86 [cf. Ibid., t. III, pl. 25], ou des artistes-commerçants : en 1882, les orfèvres Bapst et Falize choisirent la brique pour leur façade du 4-6 rue d'Antin $\left(2^{\mathrm{e}}\right)$. Ernest Lheureux travailla la surface comme une pièce d'orfèvrerie avec insertions de médaillons, camées, écus et blasons en pierre [cf. Ibid., pl. 92-93].

Enfin, si grâce aux fabricants russes et italiens et aux travaux du Nouvel Opéra, la mosaïque avait connu un grand succès en 1867, elle n'eut pas la même fortune en 1878. Gian Domenico Facchina posa au sol du palais du Trocadéro des motifs géométriques très simples, et aux murs des cartouches ornés d'inscription mais ce matériau solide et facile d'entretien ne fut réservé qu'aux pavillons publics. Pourtant, les façades parisiennes furent l'écho de cette redécouverte de la couleur et de l'insertion de matériaux diversement décoratifs. Lorsque Deslignières fut appelé dès 1880 par Louis Dormeuil pour reconstruire un théâtre à l'emplacement des Menus Plaisirs au 14, boulevard de Strasbourg $\left(10^{\mathrm{e}}\right)$, il s'entoura du mosaïste Facchina et d'artisans rencontrés au pavillon de l'Union céramique [Lœbnitz, Muller et Boulenger]. Inaugurée en avril 1881, la façade fut saluée comme un manifeste en faveur de la polychromie dans l'architecture. Á la même époque, on retrouve d'autres exemples de cette application de médaillons ou de cartouches de mosaïques sur des façades privées : à l'angle de la rue Galilée et de la rue Hamelin, Henri Fivaz et Frantz Jourdain choisirent en 1882 de traiter le pan coupé de l'immeuble comme s'il s'agissait d'un petit hôtel particulier : élevé en pierre alors que le reste du bâtiment est en briques, ses deux travées centrales reliées par une arcade cintrée encadrent un panneau à motifs d'oiseaux et de vasques; dans sa partie supérieure, un médaillon en mosaïque représente Galilée. Une frise à carreaux placée sous l'entablement reprend les teintes bleue et verte de l'ensemble [cf. fig. 6, p. 88, Ibid., t. V]. Quant à la Société pour l'instruction élémentaire, en 1880, elle éleva au 14, rue du Fouarre $\left(5^{\mathrm{e}}\right)$ un petit bâtiment : au premier étage, au dessus des fenêtres, l'architecte Roch Rozier plaça trois bandeaux de céramique à motifs floraux polychromes portant les inscriptions Arts, Morale et Sciences en lettres bleu turquoise sur un fond doré. Entre chacune d'elles, le céramiste Deck représenta en médaillons les portraits des divers présidents de la société sur fond rouge sombre : La Rochefoucauld 1818, Francour 1830, Jouard 1833 et Albert Le Roy 1867. Trois autres bandeaux de céramique à motif de coquille sur fond doré furent placés sous l'entablement. 
Les matériaux artificiels [ciments, plâtres, pierres fabriquées et bitumes] déjà présentés en abondance aux précédentes expositions nationales et internationales, s'étaient révélés particulièrement adéquats pour le Génie civil. En s'affinant, la fabrication de ces pierres artificielles permit de se substituer à la pierre de taille. En 1878, il ne s'agissait donc plus de simples placages mais de véritables blocs de béton ou de mortier de ciment moulés, qui, une fois durcis, remplaçaient avantageusement moellons et pierre de taille, garantissant durée, dureté et résistance à l'écrasement et offrant médaillons, frises courantes, et toutes sortes de motifs pour portails et façades [1'entrée principale de la Classe 66; cf. Lacroix, Études, VII, p. 312]. Le ciment Vicat fit beaucoup parler de lui car, outre sa démonstration de prise lente [coulée le $1^{\mathrm{er}}$ juin, la dalle de couvrement du pavillon fut retirée quatre semaines plus tard le 26 juin], il présentait des moulages légers à l'aspect de pierre de taille sculptée à des prix bien inférieurs. Ces préfabrications annonçaient de multiples usages en matière d'architecture et de décoration monumentale [Ibid., t. I, p. 329]. D'autres essais furent élaborés par MM. Sorel, François Coignet, et Paul-Dubos pour obtenir un béton aggloméré obtenu par un tassement du mélange et pilonné par couches successives, ou du ciment armé coulé dans une carcasse en fer [Ibid., t. I, p. 530-31] : toutes ces pierres artificielles se voulaient susceptibles de remplacer avantageusement la pierre de taille pour marches d'escalier, bordures de trottoirs, couronnement de murs mais également balustrades, portes cochères, croisées moulurées de corniches, pilastres, perrons, socles, vases. MM. Lippmann et Schneckenburger proposèrent de nouvelles variantes de similipierre où le ciment mélangé à des substances minérales réduites en poudre [albâtre, marbre, sable, brique] permettait d'obtenir un produit économique, facile à sculpter et à polir, et très résistant aux intempéries [Ibid., p. 532]. L'Autriche-Hongrie, l'Italie, la Chine et l'Angleterre présentèrent des bétons teintés dans la masse : ainsi, le système Lascelles fut employé par les anglais pour imiter la brique, rue des Nations.

Cependant, malgré la qualité de la production, la beauté de ces produits et leur bon marché relatif, Eugène Lacroix avouait une nette préférence pour la pierre [Ibid., t. VII, p. 309]. Bien que réputées et très exportées [plus de 109205 tonnes en 1876], les pierres de taille françaises ne furent utilisées qu'à de rares exceptions dans cette exposition éphémère où leur coût et leur délai d'exploitation auraient retardé considérablement la livraison. Par contre, les exploitants de carrières et tailleurs belges choisirent d'insérer pour leur pavillon les différents matériaux employés habituellement dans leurs constructions nationales. L'architecte Émile Janlet proposa donc pour la façade de la rue des Nations un véritable échantillonnage de ces variétés de pierre naturelle, alternant les noires, grises, bleues, brunes et blanches, et des marbres noirs et rouges. Le remplissage de briques et la teinte bleutée des ardoises posées en mosaïques sur le toit complétaient cette polychromie. Dans les années qui suivirent, la pierre de taille et ses parements fins resteront longtemps le symbole des constructions raffinées et volontairement « monumentalisées », même si elles étaient de petite taille [cf. les deux hôtels particuliers des 38-40, avenue Pierre-I ${ }^{\mathrm{er}}$-de-Serbie ( $8^{\mathrm{e}}$ ) élevés par les architectes Alfred Ferrot et Henri Fèvre (La Semaine des constructeurs, $1^{\mathrm{er}}$ novembre 1879) ou la série de quatre au 38-40, rue Boissière $\left(16^{\mathrm{e}}\right)$, construits par Alfred Rousseau (Ibid., 23 juillet 1881)]. 
Longtemps demeuré l'apanage des fabricants de vitraux ou d'artisanat d'art [tel Émile Gallé de Nancy qui obtint une médaille de bronze pour ses vases japonisants], le verre était de plus en plus utilisé dans la construction avec les tuiles en verre et les vitres-dalles de MM. Lémal et Riquet et de la Compagnie Saint-Gobain [cf. hôtel du Louvre ou salle des Pas-Perdus du Crédit lyonnais]. Dès 1881-82, l'architecte Édouard Corroyer utilisa pour la première fois le plancher de verres-dalles de Saint-Gobain récemment exposées dans le hall du Comptoir national d'Escompte, 14, rue Bergère $\left(9^{\mathrm{e}}\right)$ [cf. pl. 138, Dict., t. I, no 1273 ; Édouard Didron y réalisa les vitraux de la verrière, Aimé Millet la statuaire, Villeminot les ornements, Charles Lameire et Facchina les mosaïques, et l'orfèvre Christofle les lanternes extérieures]. S'il n'apparaît pas encore comme élément de décor à part entière, le verre entrait ainsi dans la construction ellemême et soignait son aspect décoratif, premier pas vers ce que seront les réalisations monumentales de René Lalique à la fin du siècle.

Grâce à la nouvelle puissance mécanique des forges, le travail du métal avait gagné en précision, vitesse et qualité : les industriels du fer et des tôles ouvrées exposèrent sur plans ou dans plusieurs bâtiments métalliques disséminés dans les jardins et les quais les avancées nouvelles de leur art [cf. le projet de Halle-Basilique de Louis Auguste Boileau (cf. Lacroix, Principes et exemples d'architecture ferronnière); cf. serres, volières et autres petits édifices (Arch. nat., $\mathrm{F}^{12} 3226$ et $\mathrm{F}^{12}$ 3518)]. Avec l'élévation constante du prix du bois et l'évolution du travail des métaux, la nouvelle profession de "ferronnier-constructeur " avait peu à peu vu le jour : en effet, le serrurier actuel fabrique non seulement nos planchers, nos combles de bâtiments, mais encore des murailles et des pans de fer; il construit les couvertures immenses des halles, des marchés, des gares de chemin de fer, les ponts tubulaires et beaucoup d'autres ouvrages très considérables [Lacroix, Études, VII, p. 282]. La diffusion croissante de pièces décoratives en métal laminé entraina la nécessité d'un traitement ornemental dont les motifs étaient posés en applique et le plus facilement du monde, c'est à dire à l'aide de vis à métaux [Ibid., IV, p. 71]. L'ornement métallique y trouva de nouvelles applications et la serrurerie traditionnelle une sorte de renaissance avec la reprise du relevage en bosse [cf. Lacroix, Rapport, Groupe III, Classe 25] : les fontes décoratives fabriquées en série sollicitaient l'intervention de sculpteurs pour les fleurons, culots, feuillages, cartouches et mascarons destinées aux croisées, grilles, rampes, escaliers, fontaines [cf. les masques de faune de la cascade du Trocadéro sculptés par Auguste Rodin]. Deux établissements s'illustrèrent particulièrement dans ces techniques, celui des frères Durenne et l'illustre maison Barbedienne. Le premier se distingua par deux fontaines placées dans les jardins du Champ de Mars, dans l'axe de l'entrée principale en venant de la Seine : la fontaine aux Tritons était librement inspirée de celle que le statuaire Jules Klagmann avait érigée square Louvois quelques années auparavant. L'autre dite fontaine aux Cariatides avait été dessinée par Auguste Bartholdi [qui présentait également la tête de la statue de la Liberté qu'il livra au port de New York en juillet 1884]. Le fondeur obtint un rappel de médaille d'or pour ces deux imposants travaux et son sculpteur d'ornements Doussamy obtint une « médaille de bronze de collaborateur ». Quant à Ferdinand Barbedienne, il s'était spécialisé dans la réduction de sculptures monumentales grâce au procédé d'Achille Collas qui permettait l'édition mécanisée à diverses échelles. Cette commercialisation abondante, très 
prisée par la bourgeoisie, requerrait dans les ateliers une équipe de sculpteurs expérimentés, habitués au travail de la pierre comme à celui du bronze : Bailly, Cauchois, Garnier ou Constant Sévin reçurent des médailles en tant que « collaborateurs » de la maison [elle-même récompensée d'une Grande médaille]. Les établissements SaintSauveur d'Arras spécialiste de métal découpé, l'atelier de $\mathrm{M}^{\mathrm{me}}$ Veuve Lelong, les zincs d'art de J. Ranvier prouvaient la compétitivité des fabriques industrielles auxquelles étaient associés des sculpteurs d'ornements ou des statuaires de renom comme Mathurin Moreau, Carrier-Belleuse, Piat ou Dumaige.

L'emploi de statuaire décorative dans l'architecture publique ou privée avait été relancé au Second Empire : sans fausser exagérément l'anatomie, les statuaires devaient cependant l'adapter aux exigences d'une sculpture monumentale soumises aux distorsions de la perspective et aux effets de distances. Édouard Didron dénonça certaines maladresses récentes dues à la transposition de modelages de terre cuite à grande échelle : ces soldats de crâne allure [les quatre soldats du pont de l'Alma], ne semblent-ils pas être le produit d'un grandissement de figurines en terre cuite faites pour une étagère? [Lacroix, Rapport, XIV, p. 48-49]. En conservant proportion et propriété du modelage initial, les statuaires conservaient parfois sur les façades de pierre la souplesse, la plasticité et la finesse de l'argile [cf. Jean-Baptiste Carpeaux au pavillon de Flore ou à l'Opéra]. Cependant, malgré ces critiques, l'excellence de la sculpture française n'avait que peu de rivale et le Catalogue officiel des récompenses fait état de 49 médailles françaises sur 82 (Classe 3) : la majorité était attribuée à des artistes reconnus et prisés des milieux officiels [Hiolle, Dubois, Crauk, Falguière, Millet, Ponscarme et Thomas pour ne citer que les médailles d'honneur et de $1^{\text {re }}$ classe]. Pourtant sur les bâtiments de l'exposition, la statuaire eut un rôle limité, alors qu'une part importante de la décoration des jardins lui revint [cf. statues des cascades, animaux de la terrasse et nombreuses figures disposées dans les allées]. $\mathrm{Au}$ palais du Champ de Mars, il semble que ce soit le plan géométrique qui ait déterminé le décor du bâtiment : l'effet monumental et rigoureux de l'édifice se lisait effectivement sur la façade sobre et régulière dont les ornements n'étaient là que pour appuyer et scander les effets. Au pied de chaque travée, une figure allégorique monumentale en plâtre annonçait le pays exposé, armoiries et drapeaux reprenant cette indication au sommet du mât correspondant. D'un bout à l'autre de la façade, costumes et attributs permettaient de les différencier et de saisir immédiatement par un signe distinctif conventionnel les symboles particuliers, propres à chaque nation. La porte monumentale du pavillon central était couronnée d'un fronton d'Henri Charles Maniglier et soutenu par deux femmes ailées se tenant la main : l'une portait le flambeau de la Connaissance, l'autre la gerbe de la Gloire, symboles de l'Angleterre et de la France [le statuaire fut nommé chevalier de la Légion d'honneur]. Au sommet, Adolphe Martial Thabard décora l'écusson des mots $P A X, R F$ et de la date 1878. Au Trocadéro, trente statues perchées sur la terrasse circulaire présentaient les domaines explorés dans l'exposition : Sciences, Arts et Applications techniques. Mais leur taille fut jugée disproportionnée par rapport à la masse du palais et ne permit pas de les admirer à leur juste valeur. La Renommée d'Antonin Mercié fondu en cuivre repoussé, d'une taille nettement plus opportune, dominait du toit l'ensemble de l'exposition [médaille d'honneur]. 
Outre cette statuaire sur pierre, les pavillons de la rue des Nations, du jardin et du parc présentèrent des ornements décoratifs architecturaux divers que le Nouvel Opéra avait remis au goût du jour : staff, mosaïque et carton-pâte, industrie absolument parisienne et dont le sort est très lié à l'industrie du bâtiment [Ibid., III p. 47]. C'est dans la Classe 18 qu'exposèrent ces modeleurs, mouleurs et sculpteurs d'ornements. Les maisons Hubert frères, Bandeville et fils, C. Hallé et P. Hamon furent toutes récompensées, et parmi leurs collaborateurs, furent cités A. Allard, J. Chéret, Chefdeville, Evrard, F. Gau, déjà inventoriés pour leurs participations à des chantiers parisiens de sculpture monumentale sur pierre [Arch. nat., $\mathrm{F}^{12} 3223, \mathrm{~F}^{12} 3233, \mathrm{~F}^{12} 3530$; Catalogue des récompenses, p. 88-97]. La Section étrangère n'était pas en reste sur ce point : pour ne citer que trois exemples, le Portugal présenta une restitution libre du portail de l'église san Jerónimo de Belem rue des Nations [exécutée sous la direction de l'architecte Jean-Louis Pascal par les ateliers parisiens Watrinelle et Germain (médaille de bronze)] et, dans les jardins du Trocadéro, l'Algérie exposa un palais inspiré de l'entrée monumentale de la mosquée de Sidi-Bou-Médine à Tlemcen et du minaret de la mosquée d'El Mansourah [dessinés par l'architecte Wable et réalisés par le céramiste Parvillée et l'atelier de plâtres Maublanc]. Quant à Georges Houstont, il reçut une médaille d'or pour l'ornementation du pavillon belge rue des Nations, dont Charles Auguste Fraikin avait exécuté quatre cariatides engainées.

Dans le palais du Champ de Mars, le porche monumental des Beaux-Arts édifié par Sédille présentait une façade de $12 \mathrm{~m}$ de haut entièrement décorée de terre cuite et de faïence : cette porte intérieure, traitée comme un portail extérieur, fut exécutée par les ateliers Lœbnitz qui offraient ici un véritable catalogue de leurs produits [médaille d'or]. La partie haute était décorée de trois tableaux dessinés par Émile Lévy et son élève Lazar Meyer L'Architecture, La Sculpture, La Peinture, surmontés d'un Apollon sur son char à quatre chevaux au milieu de sa gloire. Les terres cuites et faïences des chambranles (peintes, émaillées ou dorées) présentaient des guirlandes vertes décorées de feuillages fantaisie et des plaques en émail blanc enrubannées d'inscriptions aux lettres d'or: M. Lobnitz a débuté par un coup de maître; rien n'est plus beau que sa porte monumentale... Tout y est grandiose, de couleur sobre, harmonieuse distinguée, d'un décor élégant, pouvant braver la température humide de nos hivers [Revue générale, XLII, 1885, col. 266]. Á la fin de l'Exposition, les panneaux polychromes furent transportés au 4, rue Pierre-Levée $\left(11^{\mathrm{e}}\right)$ sur la façade de l'atelier de Lœbnitz qu'entreprit Sédille dès 1880 et l'architecte conserva un des verticaux dans la cour de son hôtel 28, boulevard Malesherbes $\left(8^{\mathrm{e}}\right)$ [le $4^{\mathrm{e}}$ panneau «les Arts du feu ou La Céramique » sera réalisé postérieurement comme enseigne de l'atelier]. Ce réemploi extérieur d'une composition décorative intérieure témoigne de l'extrême faculté d'adaptation tant technique qu'esthétique présentée par ce type de décor.

Un dernier exemple très remarqué de décoration architecturale fut celle du pavillon de la Ville de Paris : appliqués cette fois sur une ossature de fonte et de fer dressée par les établissements Eiffel, des motifs de terre cuite et de faïence émaillée furent associés à la brique sur les différentes façades. Les maisons Muller et Parvillée livrèrent de larges panneaux de terre rouge à bordure bleue [Arch. nat., $F^{12} 3248$ ]. Novateur, ce pavillon attestait que si le fer était introduit dans la construction, le décor n'en pâtissait pas forcément : cependant, certains contemporains regrettèrent cette orgie de céramique et leurs tons violents qui détruisaient l'harmonie des réminiscences de l'art gréco-romain. 
Partant d'une analyse attentive des lieux et des bâtiments, il apparut que la ville pour la première fois faisait elle-même partie intégrante de la visite. D'autre part, en arpentant palais, allées et jardins, le visiteur " parcourait le monde » et accomplissait un voyage immobile pédagogique qui lui faisait découvrir d'autres architectures et d'autres décors. Enfin, l'utilisation des techniques et matériaux modernes permit aux architectes et commanditaires de renouveler l'ornementation des façades parisiennes dans les années qui suivirent. Les nouvelles techniques révolutionnèrent l'ordre des étapes du chantier qui, au lieu d'être nécessairement successives, purent être antérieures, consécutives ou simultanées : la fabrication de céramique ou la production de poutrelles métalliques put ainsi précéder les fondations d'un bâtiment. Cette perturbation dans l'organisation logique des étapes du chantier entre architecture et décoration bouleversa la hiérarchie naturelle entre les corps de métiers et la fit évoluer. 